\title{
Classification of Ionized Photoevaporation Flows
}

\author{
W. J. Henney \\ Instituto de Astronomía, UNAM Campus Morelia, Michoacán, México
}

\begin{abstract}
From proplyds to champagne flows, spanning 4 orders of magnitude in size and density, photoevaporation flows are ubiquitous in $\mathrm{H}$ II regions. Such flows are also found in planetary nebulae, in the form of cometary knots, although in this case the flows are often "advectiondominated", unlike the "recombination-dominated" flows that predominate in $\mathrm{H}$ II regions.
\end{abstract}

The dynamic ionization balance in a photoevaporation flow can be approximately expressed by the formula $F_{*} \simeq u n_{0}+\alpha n_{0}^{2} h$, where $F_{*}$ is the ionizing photon flux incident on the outside of the flow, $n_{0}$ is the peak ionized density in the flow, $u$ is the initial velocity of the flow, $\alpha$ is the recombination coefficient, and $h\left(\simeq 0.1 r_{0}\right)$ is the effective thickness of the flow (e.g. Bertoldi 1989). From this equation, one can identify two regimes, depending on which of the two terms on the right-hand side predominates. If it is the first term, then one finds an "advection-dominated" flow, in which the majority of incident ionizing photons reach the ionization front (IF) and ionize fresh gas, whereas if the second term dominates, then one has a "recombination-dominated" flow, in which the majority of ionizing photons are used up in balancing recombinations in the flow, resulting in very few reaching the IF.

The dividing line between these two regimes is $n_{0} \simeq 4 \times 10^{18} h^{-1}$ for the common case in which $u \simeq c_{0} \simeq 10 \mathrm{~km} \mathrm{~s}^{-1}$ and $\alpha=\alpha_{\mathrm{B}} \simeq 2.6 \times 10^{-13} \mathrm{~cm}^{3} \mathrm{~s}^{-1}$. This is shown in Figure 1, together with the regions of the $n_{0}-h$ plane occupied by observed flows in H II regions and PNe. It can be seen that the majority of proplyds and other H II region flows, such as champagne flows, elephant trunks, and cometary globules, fall along a well-defined "main sequence", which lies roughly parallel to the advection/recombination dividing line, but substantially above it, inside the recombination-dominated region. The cometary knots in many $\mathrm{PNe}$, however, lie well below the line, inside the advection-dominated region.

This work is discussed more fully in Henney (2001). Applications to the Orion proplyds are presented in Henney \& Arthur (1998), Henney \& O'Dell (1999), and Henney, García-Díaz, \& Kurtz (2001). Applications to the Helix cometary knots are presented in O'Dell, Henney, \& Burkert (2000) and LópezMartín, et al. (2001).

\section{References}

Bertoldi, F. 1989, ApJ, 346, 735 


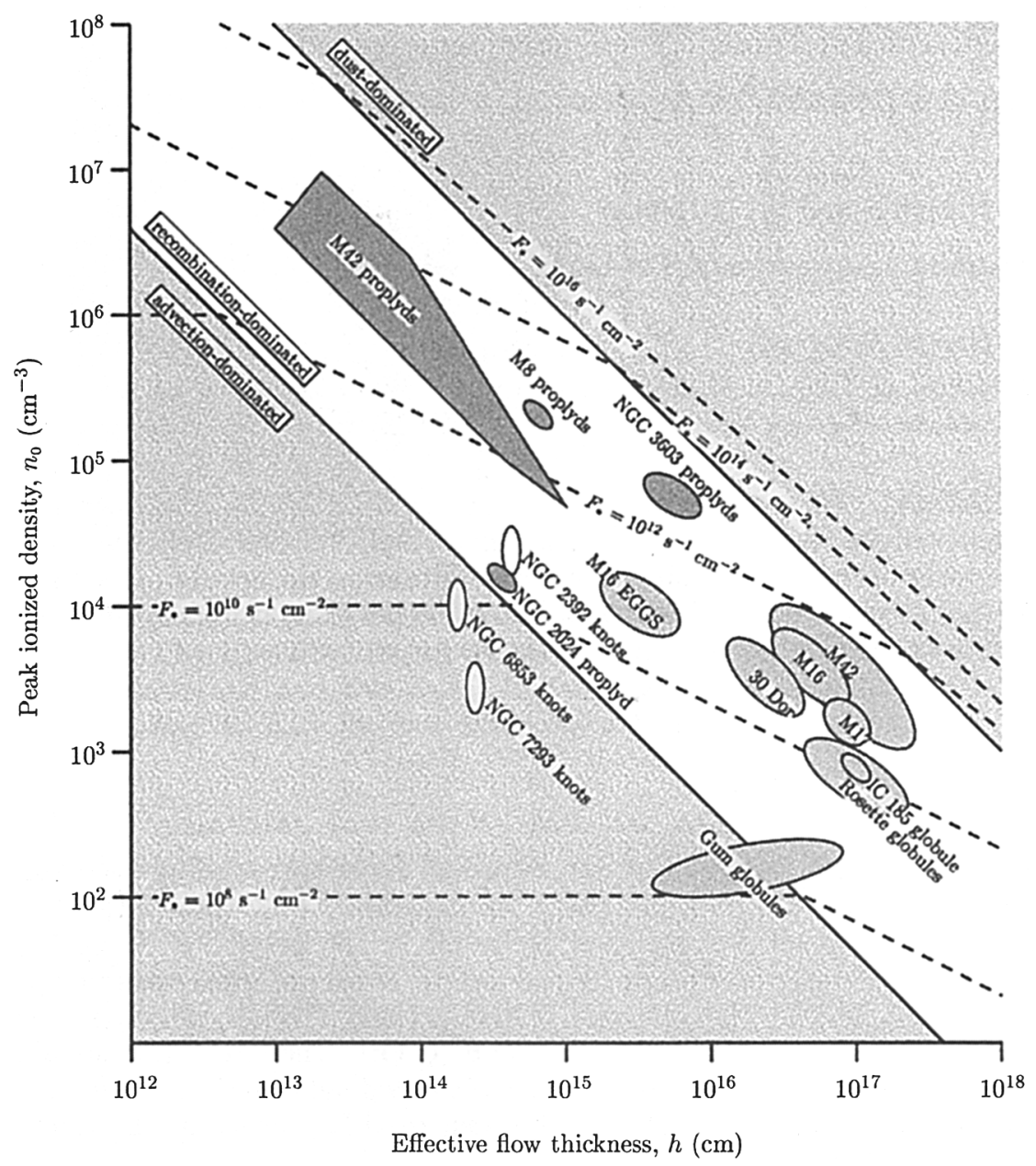

Figure 1. Peak density versus thickness for photoevaporation flows in H II regions and PNe. Shapes with the darkest shading indicate proplyds, while lighter shading shows cometary globules, elephant trunks and champagne flows. Unshaded shapes indicate PNe cometary knots. Dashed lines show contours of constant ionizing flux, $F_{*}$, and solid lines mark the boundaries between different photoevaporation regimes.

Henney, W. J. 2001, RevMexAA(SC), in press

Henney, W. J., \& Arthur, S. J. 1998, AJ, 116, 322

Henney, W. J. \& O'Dell, C. R. 1999, AJ, 118, 2350

Henney, W. J., García-Díaz, Ma. T., \& Kurtz, S. 2001, RevMexAA(SC), in press López-Martín, L., Raga, A. C., Mellema, G., Henney, W. J., \& Cantó, J. 2001, ApJ, in press

O'Dell, C. R., Henney, W. J., \& Burkert, A., 2000, AJ, 119, 2910 